Erratum

\title{
Erratum: Saastamoinen, M.; Särkijärvi, S.; Valtonen, E. The Effect of Diet Composition on the Digestibility and Fecal Excretion of Phosphorus in Horses: A Potential Risk of P Leaching? Animals 2020, 10, 140
}

\author{
Markku Saastamoinen ${ }^{1, *(\mathbb{D})}$, Susanna Särkijärvi ${ }^{1}$ (D) and Elisa Valtonen ${ }^{2}$ \\ 1 Natural Resources Institute Finland, FI-00790 Helsinki, Finland; susanna.sarkijarvi@luke.fi \\ 2 Department of Animal Science, University of Helsinki, FI-00790 Helsinki, Finland; \\ elisa.mj.valtonen@gmail.com \\ * Correspondence: markku.saastamoinen@luke.fi
}

Published: 12 February 2020

check for updates

The authors wish to make the following corrections to their paper [1]:

In Table 3, the rows showing "Intake P", "Excretion $\mathrm{P}$ " and "Digestibility $\mathrm{P}^{\prime}$ have typing errors and are now corrected (see the corrected version of Table 3 below).

Table 3. Daily intake (g), fecal excretion (g), digestibility (\%) and retention (g) of phosphorus (P).

\begin{tabular}{|c|c|c|c|c|c|c|c|c|c|c|c|c|}
\hline \multirow[b]{2}{*}{ Diet/Forage } & \multirow{2}{*}{$\begin{array}{c}\text { A } \\
\text { Hay }\end{array}$} & \multirow{2}{*}{$\begin{array}{c}\text { B } \\
\text { Haylage }\end{array}$} & \multirow{2}{*}{$\begin{array}{c}\text { C } \\
\text { Hay }\end{array}$} & \multirow{2}{*}{$\begin{array}{c}\text { D } \\
\text { Hay }\end{array}$} & \multirow{2}{*}{$\begin{array}{c}\mathrm{E} \\
\text { Hay }\end{array}$} & \multirow{2}{*}{$\begin{array}{c}\text { F } \\
\text { Hay }\end{array}$} & \multirow[b]{2}{*}{$\begin{array}{c}\text { Pooled } \\
\text { SEM }\end{array}$} & \multicolumn{5}{|c|}{ Statistical Significance ( $p$-Values) } \\
\hline & & & & & & & & $\begin{array}{c}\text { Haylage } \\
\text { vs. } \\
\text { Others }\end{array}$ & $\begin{array}{c}\text { Hay } \\
\text { vs.ConS }\end{array}$ & $\begin{array}{c}\text { Oats } \\
\text { vs.Comp }\end{array}$ & ConL & $\begin{array}{c}\text { ConT } \\
\times \\
\text { ConL }\end{array}$ \\
\hline ConL & 0 & 0 & $\mathrm{O} 20$ & $\mathrm{O} 35$ & $\mathrm{C} 20$ & $\mathrm{C} 35$ & & & & & & \\
\hline Intake $\mathrm{P}$ & 20.6 & 22.1 & 22.8 & 24.8 & 20.9 & 24.1 & 0.21 & 0.036 & $<0.001$ & $<0.001$ & $<0.001$ & 0.027 \\
\hline Excretion $\mathrm{P}$ & 20.0 & 20.2 & 21.5 & 22.1 & 19.9 & 21.5 & 0.42 & 0.125 & 0.021 & 0.025 & 0.033 & 0.251 \\
\hline
\end{tabular}

$\mathrm{O}=$ oats; $\mathrm{C}=$ complete feed; $\mathrm{ConL}=$ concentrate level $(20$ or $35 \%$ of oats $\mathrm{O}$ or complete feed $\mathrm{C})$; $\mathrm{ConS}=$ concentrate supplementation; $\mathrm{ConT}=$ concentrate type (oats/complete feed); Comp $=$ complete feed .

Conflicts of Interest: The authors declare no conflict of interest.

\section{Reference}

1. Saastamoinen, M.; Särkijärvi, S.; Valtonen, E. The Effect of Diet Composition on the Digestibility and Fecal Excretion of Phosphorus in Horses: A Potential Risk of P Leaching? Animals 2020, 10, 140. [CrossRef] [PubMed]

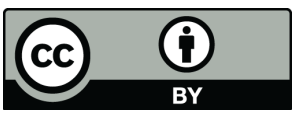

(C) 2020 by the authors. Licensee MDPI, Basel, Switzerland. This article is an open access article distributed under the terms and conditions of the Creative Commons Attribution (CC BY) license (http://creativecommons.org/licenses/by/4.0/). 